\title{
Pop-out for illusory rather than veridical trajectories with double-drift stimuli
}

\author{
Mert Özkan ${ }^{1}$ (D) $\cdot$ Peter U. Tse ${ }^{1}$ (D) Patrick Cavanagh ${ }^{1,2}$ (D) \\ Published online: 6 May 2020 \\ (C) The Psychonomic Society, Inc. 2020
}

\begin{abstract}
If a patch of texture drifts in one direction while its internal texture drifts in the orthogonal direction, the perceived direction of this double-drift stimulus (also known as the infinite regress and curveball illusions) deviates strongly from its physical direction. Here, we use double-drift stimuli to construct two types of search arrays: The first had an oddball target in terms of the physical trajectories, but no oddball for the perceived trajectory, whereas the second had a perceptual oddball, but no physical oddball. We used these two arrays to determine whether pop-out operates over physical or perceived trajectories. Participants reported the location of the odd double-drift stimulus that had either a unique physical or perceived trajectory in a set of four or eight items. When the distractors all shared one perceived trajectory, but the target had an odd perceived trajectory, it popped out even though the physical trajectories of the stimuli were mixed: Accuracy rates were at ceiling, and response times decreased with increasing set size. In contrast, participants were significantly less accurate and slower at finding the physical oddball when all the paths had a common perceived trajectory. Moreover, responses became less accurate and slower with increasing set size. Our findings suggest that, at least for this type of stimulus, perceptual features can be processed rapidly, whereas the search for physical features is very inefficient.
\end{abstract}

Keywords Visual search · Attention · Motion illusion

When a target differs from distractors by a unique, elementary feature, the stimuli are processed in parallel, and the target "pops out" exogenously, without focused attention (Treisman \& Souther, 1985). This type of processing has been termed "preattentive" and is unaffected (and sometimes even facilitated) by increasing the number of distractors (Bravo \& Nakayama, 1992). Here, we use this pop-out performance to determine whether visual search operates on the physical or perceptual properties of an illusory stimulus.

Usually, a stimulus is perceived to move in the direction that it is actually going in the world or across the retina. To answer whether pop-out occurs over perceived or actual

Electronic supplementary material The online version of this article (https://doi.org/10.3758/s13414-020-02035-w) contains supplementary material, which is available to authorized users.

Mert Özkan

mert.ozkan.gr@dartmouth.edu

1 Department of Psychological and Brain Sciences, Dartmouth College, Hanover, NJ 03755, USA

2 Department of Psychology, Glendon College, Centre for Vision Research-York University, Toronto, ON M4N3M6, Canada motion paths, it is necessary to use a stimulus that dissociates perceived and actual motion direction. The perceived path of the double-drift illusion (Lisi \& Cavanagh, 2015; Shapiro, Lu, Huang, Knight, \& Ennis, 2010; Tse \& Hsieh, 2006) deviates strongly (by $45^{\circ}$ or more) from its physical path (see Fig. 1 and Supplementary Movie 1). This allows us to create motion trajectory oddballs either among perceived or actual path orientations. A previous study showed that saccades go to the physical and not the perceived location (Lisi \& Cavanagh, 2015) of a double-drift stimulus. This raises the question of whether visual search is, like saccades, unaffected by the illusion. A few studies have examined motion trajectories in conjunction search (Frank, Greenlee, \& Tse, 2018; Reavis, Frank, Greenlee, \& Tse, 2016) or motion directions in pop-out search (Gheri, Morgan, \& Solomon, 2007; Nakayama \& Silverman, 1986; Van der Burg, Cass, \& Theeuwes, 2019), but no studies have examined motion-trajectory singletons in pop-out search.

To make the visual search stimulus, we presented an array of double-drift stimuli with multiple physical path orientations. However, we exploited the illusion to make all the different physical paths appear moving in the same perceived trajectory - say, horizontal. Among these, there was one with 


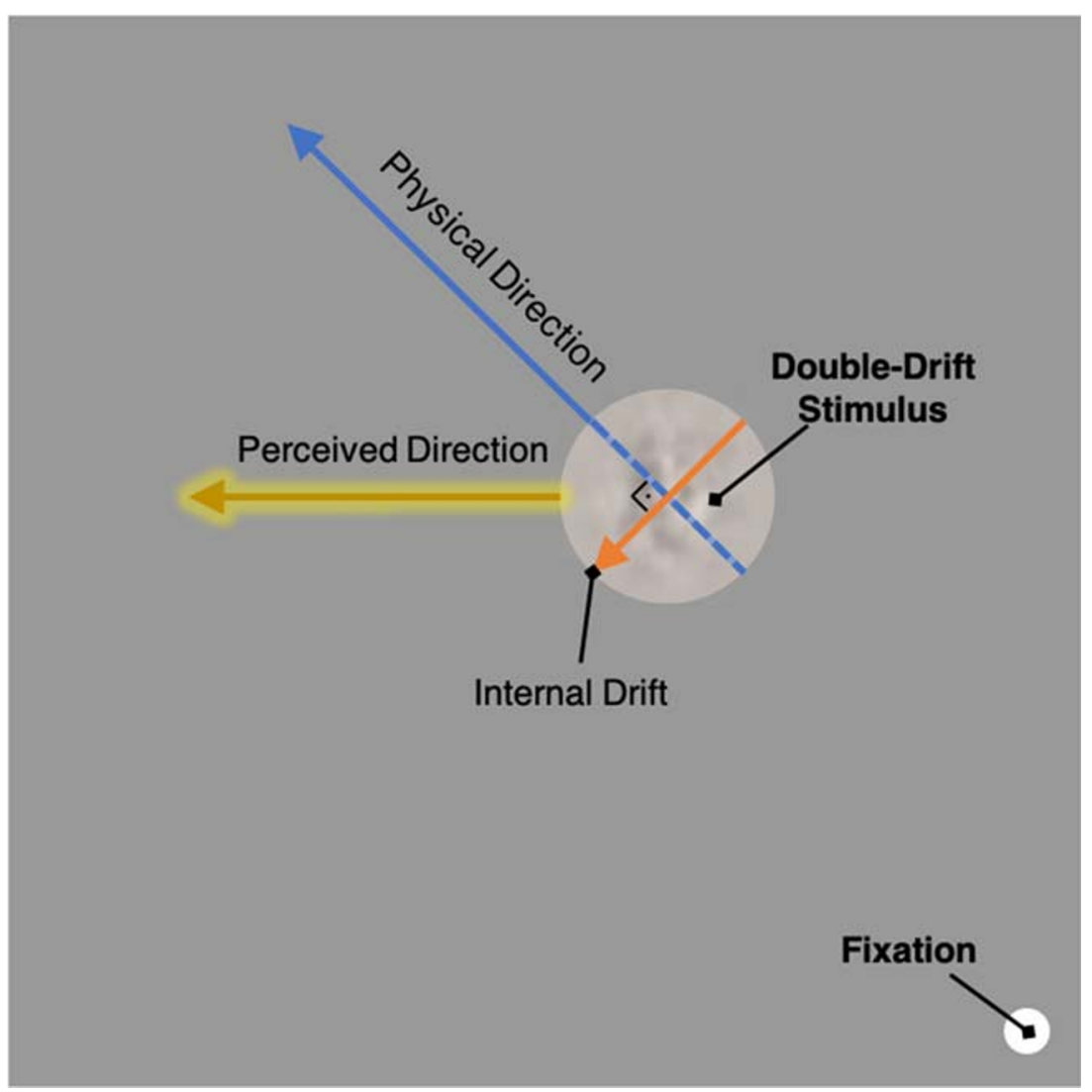

Fig. 1 Double-drift stimulus. When viewed in the periphery, a patch of random texture with internal motion orthogonal to the motion of its envelope is seen to move on a path midway between the two directions.

a different perceived trajectory — say, vertical (see Fig. 2). The odd perceived trajectory will only pop out if visual search primarily operates on perceived paths, as the physical paths have heterogeneous orientations, with no unique value. As a
The noise patch is shown lighter than the background to indicate its location. In the experiment, the noise patches always had the same mean luminance as the background

comparison, we also tested arrays that have a physical oddball, where one actual path orientation was different from the others; in contrast, exploiting the double-drift illusion again, perceived path orientations were all the same, and all differed

\section{Physical Oddball}

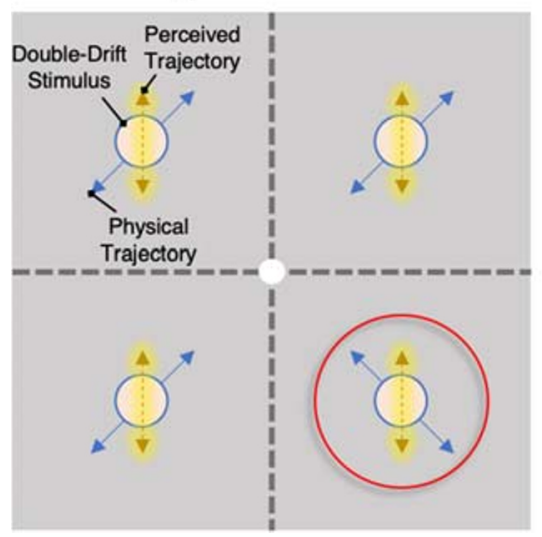

Fig. 2 Schematic of the search arrays with four items for the physical (1), perceptual (2), and control (3) pop-out conditions. In the physical case, every item followed the same perceptual trajectory, while the target was defined by its unique physical trajectory. In the perceptual case, the physical trajectories of the items were heterogeneous where every item

\section{Perceptual Oddball}

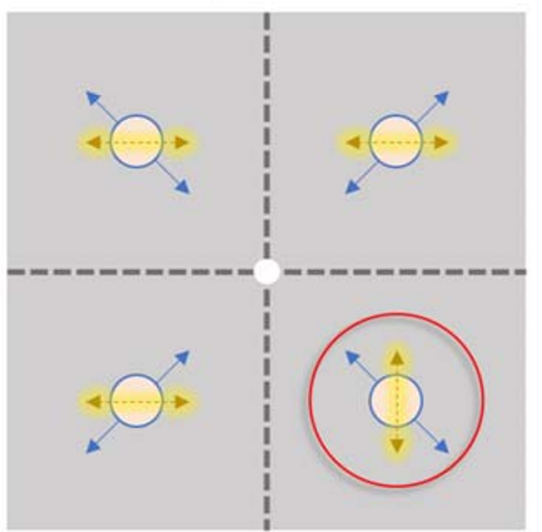

shared it with one half of the set. However, the target was defined by its unique perceived trajectory. In the control condition, the search array was identical to the physical oddball condition without consistent double-drift trajectories. Eight-item arrays were also evenly spaced and placed to avoid the meridians (highlighted by dashed gray lines) 
from the physical trajectories. If visual search operates on physical trajectories, the odd physical target should result in pop-out performance despite the uniform perceived path orientations.

Presenting multiple double-drift stimuli simultaneously in the search arrays (we used four and eight as our two set sizes) may affect the strength of the illusion. However, Haladjian, Lisi, and Cavanagh (2018) tested this and found that the illusion strength was unaffected by presenting multiple stimuli and was unaffected by attending to one or all of them. This result indicates that our procedure should be a valid test of processing of the double-drift stimulus - it does not change its characteristics when several are present at the same time. This indifference to increasing attentional load already implies that the processing of double-drift trajectories is preattentive to some extent, but it does not tell us whether visual search operates on postillusion (perceptual) or preillusion (physical) representations.

\section{Method}

\section{Participants}

Six graduate students from Dartmouth College, whose ages were between 23 and 35 years, took part in the experiment. One participant was female. All participants had normal or corrected-to-normal vision. Each participant followed the same procedure. Participants signed an informed consent approved by the Institutional Review Board at Dartmouth College. The protocol was approved by the Committee for the Protection of Human Subjects.

\section{Apparatus and stimuli}

The experiment was programmed in MATLAB R2018b using the PsychToolbox-3 (Mario et al., 2007). Testing was completed on an Apple iMac Intel Core i5 computer (Cupertino, CA). The experiment was displayed in an otherwise dark room on a 19-in. Samsung S19C200 monitor $(1,024 \times 768$ pixels at $60 \mathrm{~Hz}$ ) placed $57 \mathrm{~cm}$ from the participant. The participant's head was stabilized by a chin and forehead rest.

The central fixation target was a white circle $\left(.23^{\circ}\right.$ radius $)$. The stimuli were " $1 / \mathrm{f}$ noise" patches with a Gaussian mask ( $\sigma$ $=0.2^{\circ}$ ), which were presented in sets of four or eight. The patches had an envelope motion in one of the four possible oblique directions (i.e., $45^{\circ}, 135^{\circ}, 225^{\circ}$, and $315^{\circ}$, where $0^{\circ}$ is upward) with a speed of $4 \%$ s. The stimuli traveled back and forth along a physical trajectory of $2^{\circ}$ in length. The internal texture had a Michelson contrast of $60 \%$ and a mean that matched that of the grey background. The internal texture moved in one of the two directions orthogonal to the physical direction to create the double-drift illusion. An adjustment task was run prior to testing to determine the internal speeds required to make the oblique physical path appear horizontal or vertical. These speeds were found separately for each participant, each stimulus location, and physical trajectory. In the control condition, the internal texture moved in a random direction on each frame using the same frame-by-frame offset that matched the internal speeds of the illusory conditions (averaged over the vertical and horizontal conditions). The items were placed in isoeccentric locations, within $8^{\circ}$ away from the fixation target, while maintaining equal distance from the horizontal and vertical meridians in order to prevent the meridian effects that have been reported to interfere with the double-drift trajectory (Liu, Tse, \& Cavanagh, 2018).

In each of the three search conditions, there was one oddball (see Fig. 2). In the perceptual condition, the oddball had a unique perceived path orientation, whereas all the distractors had a common perceived trajectory that differed from that of the oddball by $90^{\circ}$. At the same time, the physical trajectories of all the items took one of two path orientations, randomly assigned (see Supplementary Movie 2). In the physical condition, the oddball had a unique physical trajectory that differed by $90^{\circ}$ from the physical trajectory of all the distractors. However, its perceived trajectory was the same as that of all the distractors (all items had a common perceived path orientation; see Supplementary Movie 3). These two conditions were compared with a control condition where there was no illusion - the internal motion randomly changed direction at each frame, thereby producing a net illusory trajectory shift of $0^{\circ}$. The trajectories in the control condition were comparable to those of the physical oddball condition with one odd physical trajectory, except that now all the perceived paths matched the physical ones so that the target was an oddball in both its physical and perceptual trajectories (see Supplementary Movie 4).

\section{Procedure and design}

Data collection was completed in five separate sessions. The first three sessions were an adjustment task where each participant adjusted the internal speed of one of the 48 stimuli (12 locations $\times 4$ physical trajectories) in order to set the perceived deviations from the oblique physical trajectory to be horizontal or vertical. They simply reported whether the stimulus moved up-down or left-right with respect to its initial position by a key specified in the instructions received every 60th trial. Thresholds were estimated using QUEST (Watson \& Pelli, 1983). Each participant ran 20 trials per condition for a total of 1,920 trials ( 12 locations $\times 4$ physical trajectories $\times 2$ perceived trajectories $\times 20$ repetitions). The average speed for the horizontal and the vertical illusions of a patch at a given location was used for the random, frame-by-frame steps of the internal texture in the control condition. 
Testing occurred in the last two sessions. Participants were instructed to press a key to start each trial once they had established visual fixation. A variable fixation interval (300$500 \mathrm{~ms}$ ) was followed by a 200 -ms-long interval during which the patches began to move. Each patch appeared and began moving at a random frame so that the motion onsets were asynchronous. This counteracted low-level cues from the internal motion of external motion directions that might have identified the pop-out target. At any given moment, some items had inward external motion, some had outward, some had clockwise, and others had counterclockwise. Similarly, the internal motion directions were shuffled by the asynchrony. This ensured that neither the internal motion nor the external motion would be a consistent cue to the perceived or physical target. Also, because the time of direction reversal was independent for each item, the items sharing external and internal drift directions changed constantly. Only the overall path orientation remained a valid cue to the target.

The stimuli kept moving until the participant responded by pressing the space bar with the left hand once he or she had localized the oddball. The timing of this key press was taken as the response time for that trial (see Fig. 3). The search display was then replaced with a response screen where each location was assigned a number (see Fig. 3, "Response Screen"). With their right hand, participants then pressed the key corresponding to the number at the location where they had seen the target. A fixation screen was displayed for at least $300 \mathrm{~ms}$ until the participants initiated the next trial.

Each trial had four or eight items on the display. Sixty-four trials per condition ( 3 oddball types $\times 2$ set sizes) were distributed randomly across two sessions.

\section{Results}

As shown in Fig. 4, left panel, participants were very accurate at reporting the oddball's location (using the location response keys; see Fig 3) in the perceptual and control conditions. Accuracy dropped markedly in the physical oddball condition, but remained above chance (which was $25 \%$ and $12.5 \%$ for the set size of four and eight, respectively). There was a significant two-way interaction between oddball type and set size, $F(2,10)=7.21, p<.02$. In particular, accuracy in the physical oddball condition dropped significantly as a function of set size, $t(5)=-4.42, p=.03$. This effect was not significant in the perceptual oddball and control conditions ( $p \mathrm{~s}>.50)$.

For reaction times, there was a significant two-way interaction between oddball type and set size, $F(2,10)=17.41, p<$ .001 . Increased set size produced faster reaction times in the perceptual oddball and the control conditions and slower reaction times in the physical oddball condition. When the perceptual oddball and control conditions were compared separately, there was a main effect of oddball type (perceptual responses were faster), $F(1,5)=14.67, p<.02$, and set size (responses for Set Size 8 were faster), $F(1,5)=13.85, p<.02$. There was no interaction $(p>.20)$.

\section{Discussion}

Our results indicate that, at least for double-drift stimuli, the oddball in perceived motion trajectory popped out. Accuracies were very high, and reaction times decreased as the set size increased, which comprises paradigmatic evidence for popout (Bravo \& Nakayama, 1992; Eymond, Cavanagh, \& Collins, 2016). This pop-out behavior was seen despite the heterogeneous orientations of the physical trajectories. The processing of the illusory array was very similar to that of the control array with a standard oddball configuration where perceptual and physical trajectories were consistent. In contrast, the physical oddball did not pop out when all the perceived paths were of the same orientation. Accuracies were much lower (although above chance), and reaction times increased with set size. When in conflict with the perceptual paths (where there was no oddball), the physical oddball could only be found with slow serial search, and even then, not very accurately.

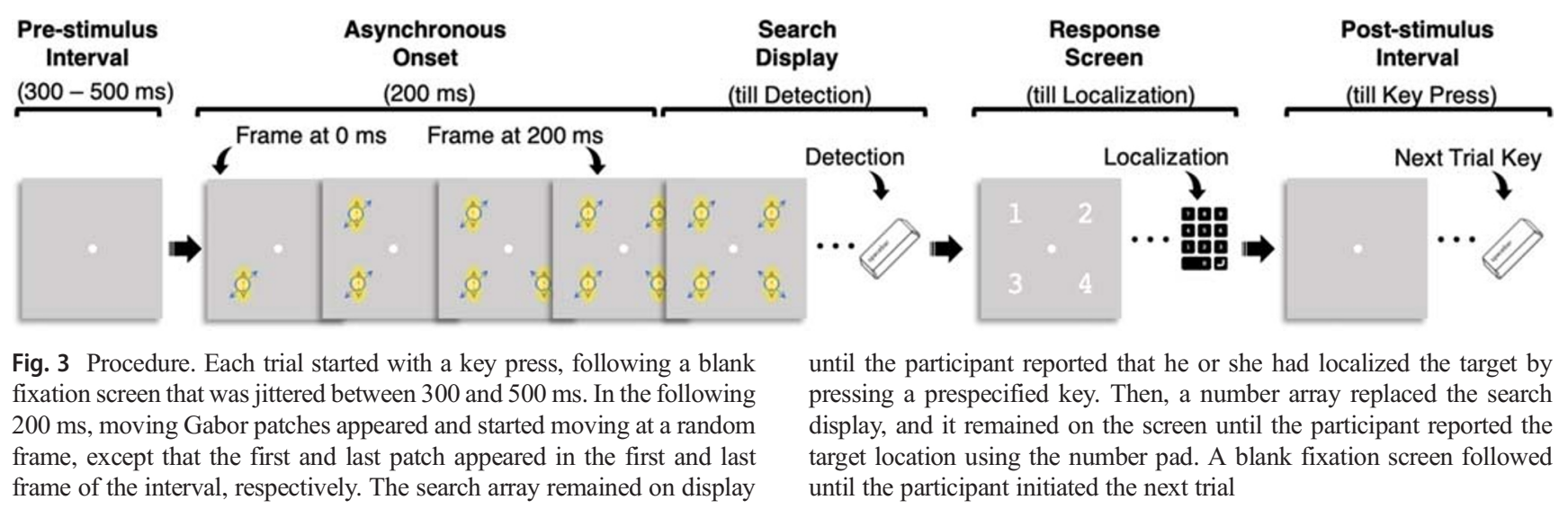




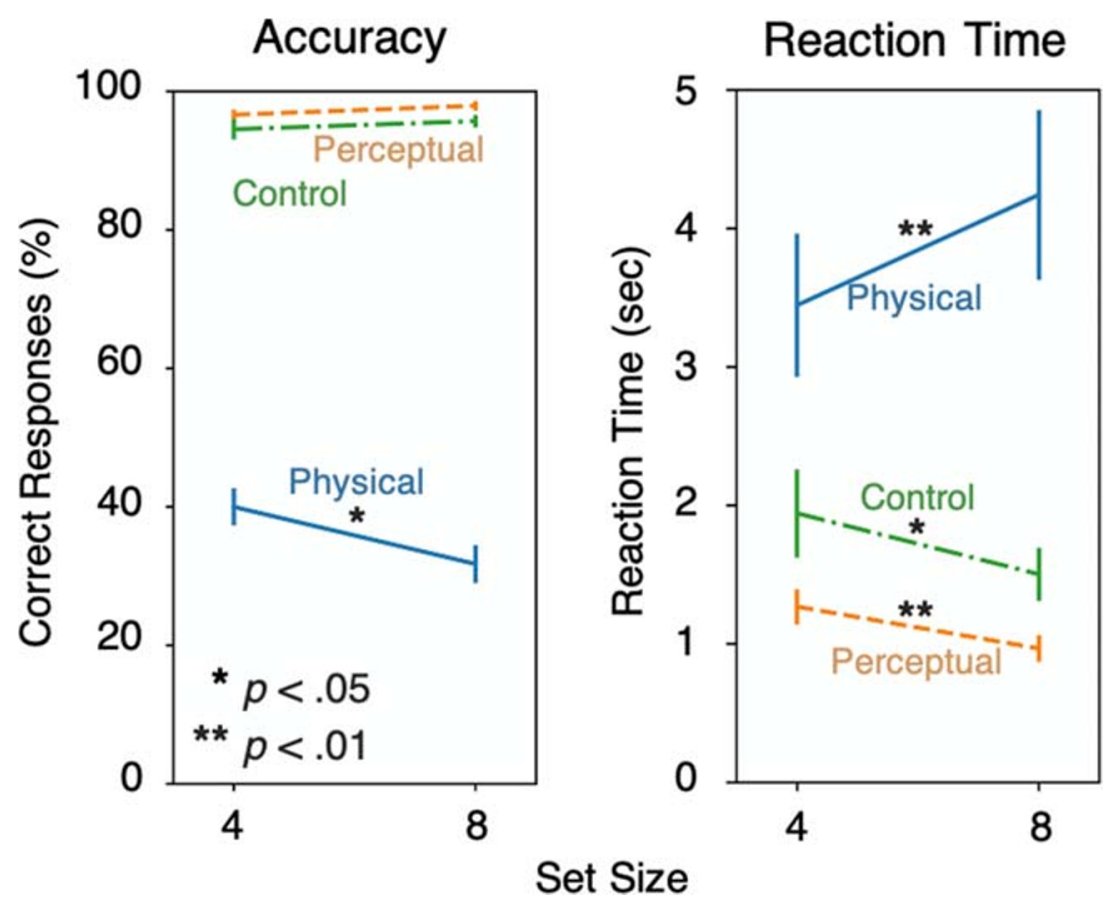

Fig. 4 Accuracy and reaction time results as a function of set size. Chance performance for accuracy is $25 \%$ for Set Size 4 and $12.5 \%$ for Set Size 8 . Significance values refer to the slopes of each function. Error bars show $\pm 1 S E$

Finally, participants were overall slower in the control condition as compared with the perceptual oddball condition. However, rather than any special speeded processing of the double-drift illusion as compared with veridical trajectories, we believe that the control stimulus itself might have caused their poorer performance. The control stimulus changed its internal drift direction randomly with each frame, which created a slight motion "wobble." Although the perceived trajectory was on average consistent with the physical trajectory, the wobble may have added noise to the orientation judgment, slowing subjects' reaction times.

Pop-out among perceived rather than physical motion paths adds to the growing list of high-level features that pop out. Beyond the initial findings of pop-out for primitive features associated with early visual cortex (orientation, color, motion, size, etc.; Treisman, 1985), several papers have reported pop-out for attributes that are clearly computed at higher levels, such as depth from shading (Enns \& Rensink, 1990; Kleffner \& Ramachandran, 1992) and shape after amodal completion (He \& Nakayama, 1992). The present study using illusory motion trajectories expands this set further. Our finding strongly suggests that the perceived doubledrift trajectory is processed efficiently in visual search, whereas the physical features (when in conflict with perceptual features) are processed very inefficiently. Our study is consistent with the predictions of the reverse hierarchy theory proposed by Hochstein and Ahissar (2002). According to these authors, high-level features are more easily accessed by visual search, while search for low-level features may require an unpacking of the higher-level description.

This lack of access to early features indicates again that we cannot see these initial representations - we cannot see our retinas (Perdreau \& Cavanagh, 2011). Visual search appears to operate at the level after correction for eye movements, distance, and object motion - after visual constancies have created a stable representation of the world (Rock, 1983). Even though the participants could not "see" the physical motion trajectories, they were able to make responses, although more slowly and less accurately. In the physical feature pop-out condition, the orientation of each item's perceived path was all the same and gave no cue to which item had the unique physical trajectory. We assume that participants eventually learned how to search for the item with the odd internal direction, the one defining feature of the odd physical item. However, internal direction is not a reliable cue in isolation because the patches reversed direction asynchronously - a downward physical target could share the internal motion direction with an upward distractor at any given moment, for example. Participants would have to monitor items over more than one reversal to be sure of its unique internal direction while moving - say, downward. Another potential explanation is that the physical oddball might have a pop-out response gain in the early visual cortex before it is overridden by the conscious perception (Burrows \& Moore, 2009; Yan, Zhaoping, \& Li, 2018) and that this property might be accessible with scrutiny. Future research should explore this possibility. 
How high level are the perceived double-drift trajectories? To a greater extent than other stimuli, the double-drift illusion creates a dramatic difference between the perception and the physical stimulus. Cavanagh and Tse (2019) showed that the double-drift illusion persists even with smooth pursuit nulling its motion on the retina. This indicates that the illusion must arise at or beyond the site where efference copy from eye movements are combined with retinal motions to recover motion in the world. Some likely candidate regions include V3A, V6, and MST (Fischer, Bülthoff, Logothetis, \& Bartels, 2012; Nau, Schindler, \& Bartels, 2018; Schindler \& Bartels, 2018). Furthermore, a multivariate pattern analysis study (Liu, Yu, Tse, \& Cavanagh, 2019) used crossclassification to decode perceived trajectories, training on physically matched nonillusory stimuli and vice versa. Interestingly, the cross-classification accuracy failed to exceed the chance level in early visual cortex (V1, V2, V3, MT+), whereas their searchlight analyses returned accuracy levels above chance in lateral and medial frontal regions. These studies imply that the site of emergence for the perceptual motion trajectory lies beyond the early visual cortex. The present finding, that illusory perceived path is a "primitive" feature that supports pop-out, indicates that the processing underlying pop-out search must also operate at a very high level, perhaps as anterior as the frontal cortex.

Author contributions P.U.T., P.C., and M.O. designed the study, and M.O., P.U.T., and P.C. wrote the manuscript; M.O. performed the experiment and analyzed the data.

Funding This research was supported by the NSF EPSCoR \#1632738 grant to P.U.T., funding from the Department of Psychological and Brain Sciences of Dartmouth College, and an NSERC Canada grant to P.C.

\section{Compliance with ethical standards}

Declaration of conflicting interests The author(s) declared no potential conflicts of interest with respect to the research, authorship, and/or publication of this article.

\section{References}

Bravo, M. J., \& Nakayama, K. (1992). The role of attention in different visual-search tasks. Perception \& Psychophysics, 51(5), 465-472. https://doi.org/10.3758/bf03211642

Burrows, B. E., \& Moore, T. (2009). Influence and limitations of popout in the selection of salient visual stimuli by area V4 neurons. The Journal of Neuroscience: The Official Journal of the Society for Neuroscience, 29(48), 15169-15177. https://doi.org/10.1523/ JNEUROSCI.3710-09.2009

Cavanagh, P., \& Tse, P. U. (2019). The vector combination underlying the double-drift illusion is based on motion in world coordinates: Evidence from smooth pursuit. Journal of Vision, 19(14), 2. https://doi.org/10.1167/19.14.2

Enns, J. T., \& Rensink, R. A. (1990). Sensitivity to Three-dimensional orientation in visual search. Psychological Science, 1(5), 323-326. https://doi.org/10.1111/j.1467-9280.1990.tb00227.x

Eymond, C., Cavanagh, P., \& Collins, T. (2016). Feature-based attention across saccades and immediate postsaccadic selection. Attention,
Perception, \& Psychophysics, 78(5), 1293-1301. :https://doi.org/ 10.3758/s13414-016-1110-y

Fischer, E., Bülthoff, H. H., Logothetis, N. K., \& Bartels, A. (2012). Human areas V3A and V6 compensate for self-induced planar visual motion. Neuron, 73(6), 1228-1240. https://doi.org/10.1016/j. neuron.2012.01.022

Frank, S., Greenlee, M., \& Tse, P. (2018). Long time no see: Enduring behavioral and neuronal changes in feature conjunction learning 3 years after training. Journal of Vision, 18(10), 289-289.

Gheri, C., Morgan, M. J., \& Solomon, J. A. (2007). The relationship between search efficiency and crowding. Perception, 36(12), 1779-1787. https://doi.org/10.1068/p5595

Haladjian, H. H., Lisi, M., \& Cavanagh, P. (2018). Motion and position shifts induced by the double-drift stimulus are unaffected by attentional load. Attention, Perception, \& Psychophysics, 80(4), 884893. https://doi.org/10.3758/s13414-018-1492-0

He, Z. J., \& Nakayama, K. (1992). Surfaces versus features in visual search. Nature, 359(6392), 231-233. https://doi.org/10.1038/ $359231 \mathrm{a} 0$

Hochstein, S., \& Ahissar, M. (2002). View from the top. Neuron, 36(5), 791-804. https://doi.org/10.1016/s0896-6273(02)01091-7

Kleffner, D. A., \& Ramachandran, V. S. (1992). On the perception of shape from shading. Perception \& Psychophysics, 52(1), 18-36. https://doi.org/10.3758/BF03206757

Lisi, M., \& Cavanagh, P. (2015). Dissociation between the perceptual and saccadic localization of moving objects. Current Biology: $C B$, 25(19), 2535-2540. https://doi.org/10.1016/j.cub.2015.08.021

Liu, S., Tse, P. U., \& Cavanagh, P. (2018). Meridian interference reveals neural locus of motion-induced position shifts. Journal of Neurophysiology, 119(6), 2091-2099. https://doi.org/10.1152/jn. 00876.2017

Liu, S., Yu, Q., Tse, P. U., \& Cavanagh, P. (2019). Neural correlates of the conscious perception of visual location lie outside visual cortex. Current Biology: CB, 29(23), 4036.e4-4044.e4. https://doi.org/10. 1016/j.cub.2019.10.033

Mario, K., David, B., Denis, P., Allen, I., Richard, M., \& Christopher, B. (2007). What's new in Psychtoolbox-3. Perception, 36(14), 1-1.

Nakayama, K., \& Silverman, G. H. (1986). Serial and parallel processing of visual feature conjunctions. Nature, 320(6059), 264-265. https:// doi.org/10.1038/320264a0

Nau, M., Schindler, A., \& Bartels, A. (2018). Real-motion signals in human early visual cortex. NeuroImage, 175, 379-387. https://doi. org/10.1016/j.neuroimage.2018.04.012

Perdreau, F., \& Cavanagh, P. (2011). Do artists see their retinas? Frontiers in Human Neuroscience, 5, 171. https://doi.org/10.3389/fnhum. 2011.00171

Reavis, E. A., Frank, S. M., Greenlee, M. W., \& Tse, P. U. (2016). Neural correlates of context-dependent feature conjunction learning in visual search tasks. Human Brain Mapping, 37(6), 2319-2330. Retrieved from https://onlinelibrary.wiley.com/doi/abs/10.1002/ hbm. 23176

Rock, I. (1983). The logic of perception. Cambridge, MA: MIT Press. Retrieved from https://philpapers.org/rec/ROCTLO

Schindler, A., \& Bartels, A. (2018). Human V6 integrates visual and extra-retinal cues during head-induced gaze shifts. iScience, 7 , 191-197. https://doi.org/10.1016/j.isci.2018.09.004

Shapiro, A., Lu, Z.-L., Huang, C.-B., Knight, E., \& Ennis, R. (2010). Transitions between central and peripheral vision create spatial/ temporal distortions: A hypothesis concerning the perceived break of the curveball. PLOS ONE, 5(10), e13296. https://doi.org/10. 1371/journal.pone.0013296

Treisman, A. (1985). Preattentive processing in vision. Computer Vision, Graphics, and Image Processing, 31(2), 156-177. https://doi.org/ 10.1016/S0734-189X(85)80004-9

Treisman, A., \& Souther, J. (1985). Search asymmetry: A diagnostic for preattentive processing of separable features. Journal of 
Experimental Psychology. General, 114(3), 285-310. https://doi. org/10.1037/0096-3445.114.3.285

Tse, P. U., \& Hsieh, P.-J. (2006). The infinite regress illusion reveals faulty integration of local and global motion signals. Vision Research, 46(22), 3881-3885. https://doi.org/10.1016/j.visres. 2006.06.010

Van der Burg, E., Cass, J., \& Theeuwes, J. (2019). Changes (but not differences) in motion direction fail to capture attention. Vision Research, 165, 54-63. https://doi.org/10.1016/j.visres.2019.09.008

Watson, A. B., \& Pelli, D. G. (1983). QUEST: A Bayesian adaptive psychometric method. Perception \& Psychophysics, 33(2), 113120. https://doi.org/10.3758/bf03202828
Yan, Y., Zhaoping, L., \& Li, W. (2018). Bottom-up saliency and topdown learning in the primary visual cortex of monkeys. Proceedings of the National Academy of Sciences of the United States of America, 115(41), 10499-10504. https://doi.org/10.1073/ pnas. 1803854115

Publisher's note Springer Nature remains neutral with regard to jurisdictional claims in published maps and institutional affiliations. 